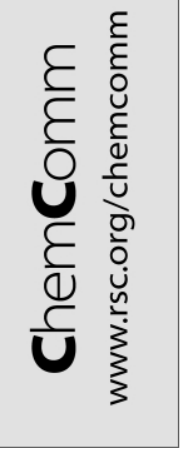

\title{
Experimental evidence for the existence of non-exo-anomeric conformations in branched oligosaccharides: the neomycin-B case $\dagger$
}

\author{
Juan Luis Asensio, ${ }^{* a}$ Ana Hidalgo, ${ }^{a}$ Igor Cuesta, ${ }^{a}$ Carlos Gonzalez, ${ }^{b}$ Javier Cañada, ${ }^{c}$ Cristina Vicent, ${ }^{a}$ \\ Jose Luis Chiara, ${ }^{a}$ Gabriel Cuevas ${ }^{c}$ and Jesus Jimenez-Barbero ${ }^{c}$ \\ a Instituto de Química Organica (CSIC), Juan de la Cierva 3, Madrid, Spain. E-mail: iqoal10@iqog.csic.es; \\ Fax: +34-91-5644853; Tel: +34-91-5622900 \\ ${ }^{b}$ Instituto de Estructura de la materia (CSIC), Madrid, Spain \\ c Centro de Investigaciones Biologicas (CSIC), Madrid, Spain
}

Received (in Cambridge, UK) 12th June 2002, Accepted 19th August 2002

First published as an Advance Article on the web 29th August 2002

For the first time in natural O-glycosides, a large amount of non-exo-anomeric conformation is experimentally detected, in solution

It is usually assumed that the exo-anomeric effect is a major factor governing the conformational behavior of natural oligosaccharides. ${ }^{1-2}$ Conformational flexibility in these molecules mainly concerns the aglyconic $\psi_{(\mathrm{C} 1-\mathrm{O} 1-\mathrm{Cx}-\mathrm{Hx})}$ angle since $\phi_{\text {(H1-C1-O1-Cx) }}$ is restricted by this stereoelectronic effect. ${ }^{3-4}$ In fact, to the best of our knowledge there is no reported case of a natural glycoside adopting a non-exo-anomeric conformation in solution. In addition, regarding flexibility, within natural sugars, branched type oligosaccharides including sugar residues glycosidated at contiguous positions (as blood type carbohydrate antigens i.e., Lewis $\mathrm{X}$ ) have been considered as the paradigm of rigid saccharides, the rigidity enhanced by van der Waals interactions. ${ }^{5-6}$ Herein, we unambiguously demonstrate that both common beliefs are not general. In neomycin-B (Fig. 1), a branched oligosaccharide antibiotic, for the first time in natural sugars, a large amount of non-exo-anomeric conformation is experimentally detected, in solution. Moreover, this unusual behavior is related to the existence of branching. Here, polar contacts between non-vicinal sugar units leads to an enhanced flexibility of the ribose glycosidic torsion $\phi$.

As a first step, in our structural analysis of neomycin-B, selective 1D-NOE experiments were carried out at $313 \mathrm{~K}(\mathrm{pH}$ 4.7). The branched nature of neomycin-B allowed the measurement of an unusually large number of structurally relevant NOEs (Fig. 1). In addition ${ }^{3} J$ values were measured for the ribose ring. The analysis of the couplings for the idose ring unambiguously show that the ${ }^{1} C_{4}$ (L) conformer (with three axial and two equatorial substituents) is by far the major one in water (>98\%). This observation is biologically relevant: the reported X-ray conformation for this ring in the ribosome- a)

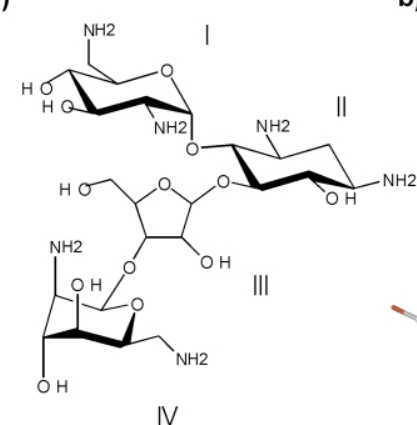

b)

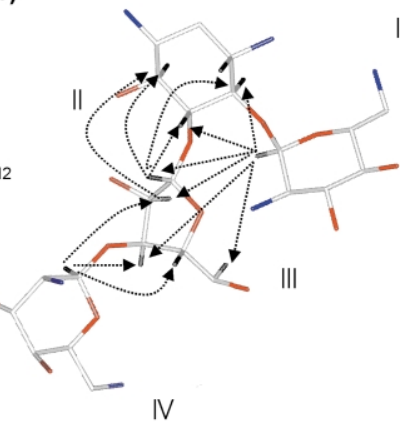

Fig. 1 (a) Structure of neomycin-B along with the numbering employed for the different sugar units. (b) Schematic representation of the measured NOEs at $\mathrm{pH} 4.7$ and $313 \mathrm{~K}$. Oxygen and nitrogen atoms are shown in red and blue respectively. Only those protons involved in relevant NOE contacts are shown (in black).

$\dagger$ Electronic supplementary information (ESI) available: experimental and theoretical parameters; Figs. S1-7. See http://www.rsc.org/suppdata/cc/b2/ b205566j/ bound state of paromomycin (an antibiotic of the neomycin-B family with 2-amino-2-deoxy-D-glucose instead of 2,6-diamino-2,6-dideoxy-D-glucose) is the opposite ${ }^{4} C_{1}(\mathrm{~L})$, with two axial and three equatorial substituents. ${ }^{7}$

In order to get an experimentally derived ensemble, $80 \mathrm{~ns}$ MD-tar ${ }^{8}$ (molecular dynamics simulations with time-averaged restraints) simulations were carried out by including $5 \mathrm{Js}$ (Table 1) and 14 experimental distances as time-average restraints with AMBER 5.0. The obtained distribution (in vacuo, $\varepsilon=80$ ) of conformers for every particular glycosidic linkage is shown in Fig. 2, superimposed on the steric MM3* maps. It can be observed that for the 2,6-diamino-2,6-dideoxy-D-Glc $\alpha$ $(1 \rightarrow 4)$-2-deoxy-Strp $\ddagger$ linkage (Fig. 1, rings I and II) a major population is centered around $\phi / \psi=-60^{\circ} \%-40^{\circ}$. A very minor population around $\phi / \Psi=-25^{\circ} / 40^{\circ}$ was also detected. In contrast, the 2,6-dideoxy-2,6-diamino-L-Ido $\beta(1 \rightarrow 3)$-Rib linkage (rings III and IV) is characterized by a high degree of flexibility with two different conformations, almost equally populated, located at $\phi / \Psi=-55^{\circ} /-50^{\circ}$ and $\phi / \Psi=-55^{\circ} / 40^{\circ}$. Nevertheless, both glycosidic linkages exhibit common features: $\phi$ values are scattered around the exo-anomeric region and conformational flexibility is mainly restricted to the aglyconic

Table 1 NOE derived distances and coupling constant values measured for neomycin $\mathrm{B}$ at $313 \mathrm{~K}$ and $\mathrm{pH}$ 4.7. Average distances obtained (from left to right) from unrestrained MD simulations, from MD-tar runs including only coupling constants, and from MD-tar simulations using $J$ and distances are shown for comparison

\begin{tabular}{|c|c|c|c|c|}
\hline${ }^{a} d(\AA)$ & Exp & MD & MD-tar $J$ & MD-tar \\
\hline $\mathrm{H} 1_{\mathrm{glc}}-\mathrm{H} 4_{\text {strp }}$ & 2.5 & 2.7 & 2.8 & 2.6 \\
\hline $\mathrm{H} 1_{\mathrm{glc}}-\mathrm{H} 5_{\mathrm{strp}}$ & 3.0 & 3.0 & 2.9 & 3.1 \\
\hline $\mathrm{H} 1_{\mathrm{glc}}-\mathrm{H} 3_{\text {strp }}$ & - & 4.4 & 4.3 & 4.3 \\
\hline $\mathrm{H} 1_{\mathrm{glc}}-\mathrm{H} 5_{\mathrm{rib}}$ & 3.0 & 3.2 & 4.5 & 3.1 \\
\hline $\mathrm{H} 1_{\mathrm{glc}}-\mathrm{H} 1_{\text {rib }}$ & 3.9 & 3.9 & 4.0 & 3.7 \\
\hline $\mathrm{H} 1_{\mathrm{glc}}-\mathrm{H} 2_{\text {rib }}$ & 3.6 & 3.1 & 3.1 & 3.4 \\
\hline $\mathrm{H} 1_{\mathrm{glc}}-\mathrm{H} 3_{\mathrm{rib}}$ & $>3.5$ & 3.6 & 3.2 & 3.1 \\
\hline $\mathrm{H} 1_{\text {rib }}-\mathrm{H} 5_{\text {strp }}$ & 2.3 & 2.5 & 2.6 & 2.5 \\
\hline $\mathrm{H} 1_{\text {rib }}-\mathrm{H} 4_{\text {strp }}$ & $>4.0$ & 4.5 & $>4.0$ & 4.4 \\
\hline $\mathrm{H} 1_{\text {rib }}-\mathrm{H} 6_{\text {strp }}$ & 3.6 & 3.3 & 3.2 & 3.3 \\
\hline $\mathrm{H} 2_{\text {rib }}-\mathrm{H} 6_{\text {strp }}$ & 3.3 & 3.3 & 3.3 & 3.4 \\
\hline $\mathrm{H} 1_{\text {rib }}-\mathrm{H} 4_{\text {rib }}$ & 3.2 & 3.1 & 3.1 & 3.1 \\
\hline $\mathrm{H} 1_{\text {ido }}-\mathrm{H} 3_{\text {rib }}$ & 2.6 & 2.7 & 2.7 & 2.5 \\
\hline $\mathrm{H} 1_{\text {ido }}-\mathrm{H} 2_{\text {rib }}$ & 3.1 & 3.0 & 2.7 & 3.0 \\
\hline $\mathrm{H} 1_{\text {ido }}-\mathrm{H} 4_{\text {rib }}$ & 3.9 & 4.1 & 4.3 & 3.7 \\
\hline$J(\mathrm{~Hz})$ & Exp & MD & MD-tar $J$ & MD-tar \\
\hline $\mathrm{H} 1_{\text {rib }}-\mathrm{H} 2_{\text {rib }}$ & 2.7 & 4.3 & 3.2 & 3.2 \\
\hline $\mathrm{H} 2_{\text {rib }}-\mathrm{H} 3_{\text {rib }}$ & 4.5 & 4.0 & 4.3 & 4.0 \\
\hline $\mathrm{H} 3_{\mathrm{rib}}-\mathrm{H} 4_{\text {rib }}$ & 6.3 & 4.6 & 6.1 & 5.8 \\
\hline $\mathrm{H} 4_{\text {rib }}-\mathrm{H} 5^{\mathrm{R}}$ rib & 5.5 & 4.5 & 2.1 & 5.2 \\
\hline $\mathrm{H} 4_{\text {rib }}-\mathrm{H} 5_{\text {rib }}^{\mathrm{sib}}$ & 3.2 & 3.2 & 2.8 & 3.8 \\
\hline
\end{tabular}

$a$ Unrestrained simulations and MD-tar runs including only $J$ values were carried out using explicit solvent, periodic boundary conditions, and Ewald sums for the treatment of electrostatic interactions. Restrained MD-tar simulations including NOE and $J$ information were performed in vacuo and with $\varepsilon=80$. 

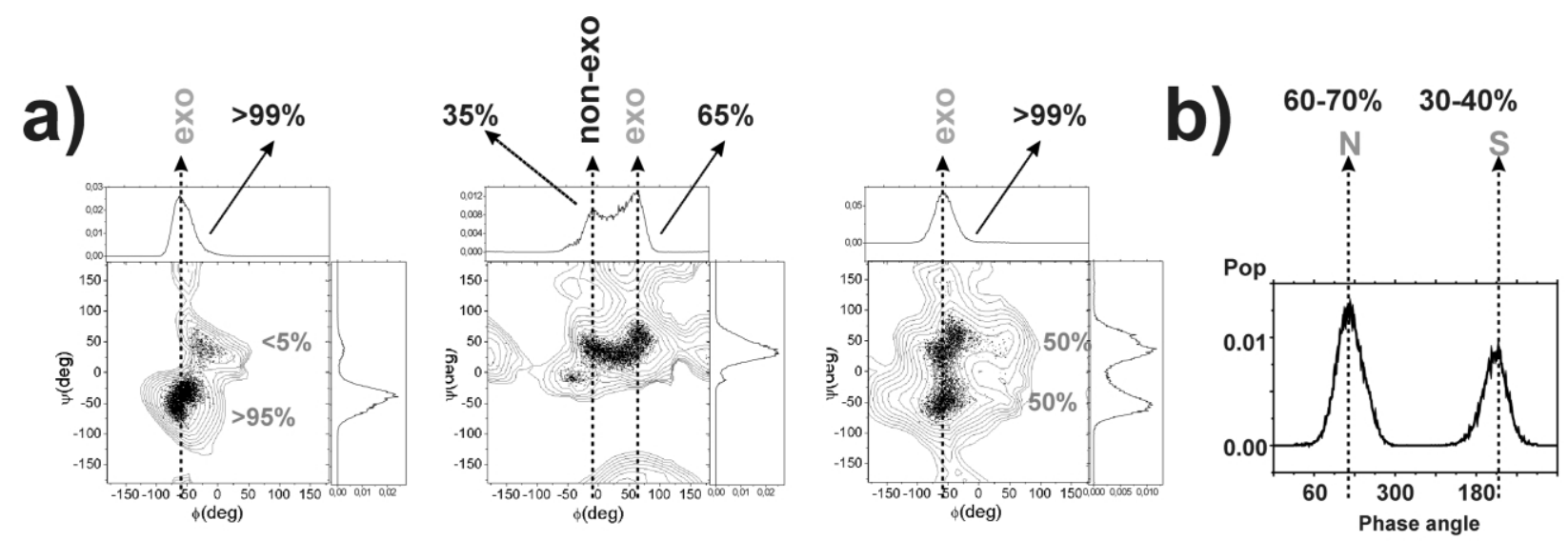

Fig. 2 (a) Experimentally derived MD-tar distributions obtained from a 80 ns length simulation for the Glc/Strp (left), Strp/Rib (middle) and Rib/Ido (right) linkages. The presence of a very significant non-exo-anomeric population for the Strp/Rib linkage (middle) is highlighted in black. (b) Ribose puckering distribution from MD-tar simulations.

$\psi$ torsion. This constitutes the usual behavior for all natural $\mathrm{O}$ glycosides described so far. A totally different behavior is observed for the $\operatorname{Rib} \beta(1 \rightarrow 5)$-2-deoxy-Strp linkage (rings II and III). In this case, two populations characterized by different $\phi$ values $\left(60^{\circ} / 45^{\circ}\right.$ and $\left.-10^{\circ} / 35^{\circ}\right)$ were detected. In contrast to common behaviour, this linkage shows a larger degree of mobility around $\phi$ than around $\psi$. Moreover, MD-tar simulations indicate that a large percentage of the population $(30 \%)$ is located in non-exo-anomeric regions, with $\phi c a .-10^{\circ}$. The nonexo-anomeric orientation of $\phi$ is experimentally detected by a medium $\mathrm{NOE} \mathrm{H} 2_{\mathrm{Rib}}-\mathrm{H} 6_{\text {Strp }}$, exclusive of this conformational region, that is, the existence of a short $\mathrm{H}-2_{\mathrm{Rib}} / \mathrm{H}-6_{\text {Strp }}$ average distance can never be explained without assuming a remarkable deviation from the exo-anomeric region for $\phi_{\mathrm{Rib}}\left(\right.$ the $\mathrm{H}-2_{\mathrm{Rib}} / \mathrm{H}-$ $6_{\text {Strp }}$ distance for the exo-anomeric region is longer than $4.1 \AA$ ). This NOE was detected under a variety of temperature conditions for neomycin-B. Moreover, it is even stronger for ribostamycin (the branched trisaccharide fragment of neomycin-B).

It is important to bear in mind that despite the relatively large number of experimental constraints employed (two or three times the usual number in sugars), the system has still a certain degree of underdetermination. This is a normal problem when flexibility is considered in the interpretation of NMR data for carbohydrates. Therefore, in principle, some dependency of the conformational populations on the computational details would be expected. However, this dependency can be assessed by performing the MD simulations under a variety of different conditions. For neomycin-B, MD simulations were carried out: unrestrained, including only $J$ information, and including both $J$ and NOE data. In the three cases, trajectories were also collected: without and with charges (with both $\varepsilon=80$ and $\varepsilon=$ $4 *$ r). Finally, unrestrained MD runs and those including only $J$ values as restraints were also performed with explicit solvent, periodic boundary conditions, counter ions and Ewald sums for the treatment of the electrostatic interactions. Average distances and $J$ values were calculated from all these trajectories and compared with the experimental ones. For the unrestrained calculations, and those including only $J$ information, a qualitative agreement between the theoretical and experimental $J$ and NOE data (including the non-exo-anomeric $\mathrm{H} 2 \mathrm{Rib}_{\mathrm{R}}-\mathrm{H} 6_{\mathrm{Strp}}$ distance) was obtained only when explicit solvent was employed. In contrast, MD-tar simulations including both NOE and $J$ information were found to quantitatively reproduce all the data, independently of the simulation conditions. More important, in all cases the conformational behavior predicted for neomycin-B was very similar. Although some differences in the obtained distributions for $\phi_{\text {Rib }}$ were observed, all these calculations showed the existence of remarkable deviations from the exo-anomeric region. The simulations carried out with explicit solvent (either unrestrained or including only $J \mathrm{~s}$ ) predicted nonexo-anomeric populations for $\phi_{\text {Rib }}$ very similar to those given by the in vacuo trajectories that included both NOEs and $J$ s. This effect has its origin in the existence of polar contacts between the non-vicinal Glc and Rib moieties. In fact, solvated MD-tar simulations carried out in identical conditions for the nonbranched 2,6-dideoxy-2,6-diamino-L-Ido $\beta(1 \rightarrow 3) \operatorname{Rib} \beta(1 \rightarrow 5)$ 2-deoxy-Strp fragment of neomycin-B (rings II, III and IV) conclusively show that the overall rigidity around $\phi_{\mathrm{R} i b}$ increases and now all torsion values are consistent with the exo-anomeric effect.

Comparison of all these data clearly indicates that a $60-70 \%$ $\mathrm{N}$ (phase angle of $7^{\circ}$ ), 40-30\% $\mathrm{S}$ (phase angle of $155^{\circ}$ ) puckering distribution for the ribose ring, together with a significant non-exo-anomeric (between $10^{\circ}$ and $-30^{\circ}$ ) population for $\phi_{\text {Rib }}(20-30 \%$, depending on the conditions) are indeed essential to satisfy the $\mathrm{H} 2_{\mathrm{Rib}}-\mathrm{H} 6_{\text {Strp }}$ NOE. This conclusion is not based on the analysis of a single MD trajectory, but on the extensive comparison of the results obtained under different simulation conditions. In this sense, the results are relatively independent of the particular details of the calculations.

Some differences in the puckering distribution of the ribose ring in neomycin-B can be observed in comparison to results based on ab initio calculations on methyl $\beta$-D-ribofuranoside reported by Serianni et al. ${ }^{9}$ Probably, the different pattern of substitution of the furanose ring, solvation effects and nonvicinal polar contacts between the glucose and ribose unit in neomycin are at the origin of these differences.

Our results indicate that flexibility in natural O-glycosides is not exclusively restricted to the aglyconic $\psi$ angle, but in certain cases $\phi$ can also undergo conformational fluctuations even adopting non-exo-anomeric orientations. In neomycin-B, rigidification of $\psi_{\text {Rib }}$ angle is compensated by remarkable enhancement of the internal mobility around $\phi_{\text {Rib }}$.

We thank BQU2000-C1501 and BQU2001-3693 for support.

\section{Notes and references}

$\ddagger$ The glucose, streptamine, ribose and idose moieties will be referred as Glc, Strp, Rib and Ido respectively.

1 E. Juaristi and G. Cuevas, The anomeric effect, 1994, CRC Press, Boca Raton, Florida, USA.

2 I. Tvaroska and T. Bleha, Adv. Carbohydr. Chem. Biochem., 1989, 47, 45.

3 T. Peters and B. M. Pinto, Curr. Opin. Struct. Biol., 1996, 6, 710.

4 J. O. Duus, C. H. Gotfredsen and K. Bock, Chem. Rev., 2000, 100 , 4589.

5 H. F. Azurmendi and C. A. Bush, Carbohydr. Res., 2002, 337, 905.

6 C. A. Bush, Z. Y. Yang and N. B. N. Rao, J. Am. Chem. Soc., 1986, 108, 6168.

7 A. P. Carter, W. M. Clemons, D. E. Brodersen, R. J. Morgan-Warren, B. T. Wimberly and V. Ramakrishnan, Nature, 2000, 407, 340.

8 D. A. Pearlman, J. Biomol. NMR, 1994, 4, 1.

9 C. A. Podlasek, W. A. Stripe, I. Carmichael, M. Shang, B. Basu and A. S. Serianni, J. Am. Chem. Soc., 1996, 118, 1413-1425. 